\title{
Fairness-Aware Throughput Maximization for Underlaying Cognitive NOMA Networks
}

\author{
Lei Xu IEEE Member, Hong Xing IEEE Member, Yansha Deng IEEE Member, A. Nallanathan IEEE Fellow, \\ and Chenlu Zhuansun
}

\begin{abstract}
To improve the radio spectral efficiency for 5G and beyond, novel radio access techniques need to be designed to accommodate unprecedented number of connected devices, and non-orthogonal multiple access (NOMA) has become a promising candidate. Additionally, power allocation and NOMA-secondary user(SU) assignment technology is an efficient way to enhance the resource utilization efficiency at the power domain and the spectral domain for underlaying cognitive NOMA networks. In this paper, firstly, a joint power allocation and SU assignment problem is formulated for NOMA downlink transmission in an underlaying cognitive radio network. The worst-case achievable rate for the NOMA-SU is maximized. To solve this mixedinteger non-linear programming (MINLP) problem, we divide the original optimization problem into two sub-problems: NOMA-SU assignment and power allocation. Next, a heuristic algorithm is adopted to solve the NOMA-SU assignment subproblem, and successive convex approximation (SCA) based method is utilized to design a suboptimal power allocation algorithm. Furthermore, an alternative joint NOMA-SU assignment and power allocation scheme is proposed with its average computational complexity analysis given. Finally, numerical results show that the total throughput for the proposed algorithm outperforms more than 30 percent compared with an existing benchmark scheme at least.
\end{abstract}

Index Terms-underlaying NOMA networks, NOMA-SU assignment and power allocation, heuristic algorithm, successive convex approximation.

\section{INTRODUCTION}

With the upsurge of the number of wirelessly connected devices, radio communication technology is evolving into $5 \mathrm{G}$ and beyond to provide greater spectral efficiency, ultra-high reliability, ultra-low latency, and higher connectivity [1], [2]. For each generation of wireless networks, multiple access technology is the key feature, e.g., code division multiple access (CDMA) for the 3G, and orthogonal frequency division multiple access (OFDMA) for the $4 \mathrm{G}$ mobile networks. As a 5G-enabling technology, non-orthogonal multiple access (NOMA) leverages the interference cancellation technique to improve the spectral efficiency [3], [4]. For example, superposition coding (SC) can be adopted at the transmitter to exploit the power-domain multiplexing, and the signals at the receiver

Lei $\mathrm{Xu}$ and Chenlu Zhuansun are with the School of Computer Science and Engineering, Nanjing University of Science and Technology, Nanjing, China. (E-mail: xulei_marcus@126.com).

Hong Xing is with the College of Information Engineering, Shenzhen University, Shenzhen 518060 China. (E-mail: hong.xing@szu.edu.cn).

Yansha Deng is with Department of Informatics, Kings College London, United Kingdom (E-mail: yansha.deng@kcl.ac.uk).

A. Nallanathan is with the School of Electronic Engineering and Computer Science, Queen Mary University of London, London E1 4NS, U.K. (E-mails: a.nallanathan@qmul.ac.uk). of each mobile terminal are separated by successive interference cancellation (SIC). Additionally, cognitive radio utilizing vacant spectrum holes has been a well-studied technology to alleviate spectrum scarcity in licensed bandwidth [5]-[7]. As a result, the underlaying cognitive NOMA networks present a promising solution for further improving the spectral efficiency of $5 \mathrm{G}$ and beyond [8].

The power allocation and NOMA-SU assignment is an important technology for underlaying cognitive NOMA networks, which can improve the throughput and fairness. In underlaying cognitive NOMA networks, the network manager allocates the power based on channel condition and optimization criterion to obtain the maximum profit, while assigns different NOMA-SUs to different subchannels to utilize the spectrum domain and power domain at the same time. Many existing references investigate the power allocation and user assignment to prove it as the effective technology for wireless NOMA networks, e.g., [9]-[18].

In this work, we investigate the joint power allocation and NOMA-SU assignment problem for underlaying cognitive NOMA networks. The contributions are summarized as follows. (i) The joint NOMA-SU assignment and power allocation problem is formulated as a mixed integer non-linear programming (MINLP) to maximize the minimum achievable rate among different SUs. (ii) Leveraging a heuristic algorithm and successive convex approximation method, an iterative joint power allocation and NOMA-SU assignment algorithm is proposed to solve the problem. (iii) The average computational complexity is analyzed for the proposed algorithms.

The rest of this work is organized as follows. We provide an overview of the related work in section II. Section III describes the network model and formulates the joint power allocation and NOMA-SU assignment problem. The joint power allocation and NOMA-SU assignment algorithm is proposed in Section IV. Sections V presents performance evaluation. Finally, Section VI concludes the paper.

\section{RELATED WORK}

Existing studies on transmission designs and resource allocations for cellular network based NOMA can be divided into two categories in terms of the optimization objective of the corresponding problems: the spectrum efficiency maximization [9]-[13] and the energy efficiency maximization [14]-[18]. For maximizing the spectrum efficiency, a global optimal solution of joint user scheduling and power allocation was presented based on mixed integer optimization for NOMA-based cellular networks [9]. In [10], a deep reinforcement learning 
framework according to attention-based neural network was investigated to support high throughput for the multi-carrier NOMA-based cellular networks. A joint power allocation and splitting control algorithm, whilst satisfying the minimum rate and the harvested energy requirements of each user, was designed to maximize the spectral efficiency for simultaneous wireless information and power transfer (SWIPT)-enabled NOMA-based cellular networks [11]. The resource allocation problem using matching theory was studied to strike a balance between throughput and fairness for NOMA-based cellular networks in centralized and distributed antenna systems [12]. The proportional fairness scheduling algorithm under imperfect channel state estimation was studied with the objective of achieving high throughput for NOMA-based cellular networks [13]. For improving the energy efficiency, a beamforming matrix with power allocation strategy subject to certain quality of service requirements was designed to minimize the total transmit power for NOMA-based cellular networks [14]. In [15], a joint dynamic power control and user pairing algorithm was proposed for power-efficient and delay-constrained hybrid NOMA cellular networks. In [16], a joint power allocation and time switching (TS) control scheme in a TS-based SWIPT NOMA-based cellular networks was investigated leveraging a dual-layer algorithm for energy efficiency maximization. In [17], a subchannel allocation and power allocation algorithm for downlink NOMA-based cellular heterogeneous networks was proposed to maximize the sum energy efficiency of the system including the macrocell and small cells. An energyefficient resource allocation problem for a machine-to-machine enabled NOMA-based cellular networks was investigated in [18] taking nonlinear energy harvesting into account.

Since cognitive radio can further improve the spectral utilization efficiency for cellular networks, resource allocation problems for cognitive NOMA networks were studied in [19]-[21]. Aiming for optimizing the user pairing and power allocation between weak users and strong users, a distributed matching algorithm subject to the users' targeted rate requirements was proposed in downlink cognitive NOMA networks in [19]. In [20], a novel dynamic power allocation scheme by flexibly meeting various quality of service requirements was designed for downlink and uplink cognitive NOMA networks in two-user cases. An optimal resource allocation approach was designed to achieve the maximum secondary throughput for energy harvesting enabled cognitive NOMA networks [21].

Although [19]-[21] studied the resource allocation algorithms for cognitive NOMA networks, the fairness in terms of the worst-case achievable rate among secondary users was not well investigated. The fairness in underlying cognitive NOMA networks is defined by the worst-case achievable rate among secondary users. If the fairness in underlying cognitive NOMA networks is high, the worst-case achievable rate among secondary users is large; otherwise, the worst-case achievable rate among secondary users becomes small. It has thus yet to be understood how to guarantee the worst-case performance of a NOMA-user whilst benefiting from multiplexing gains in a multi-user NOMA systems. In this work, we investigate the joint optimization of power allocation and NOMA-SU assignment to achieve the maximum worst-case user through- put among all the secondary users for underlaying cognitive NOMA networks.

\section{System Model AND Problem Formulation}

In this section, first, the system model for a secondary NOMA networks is introduced, composed of the transmission model, the interference temperature model, and the SU's achievable rate. Next, the joint NOMA-SU assignment and power allocation problem is formulated aiming for maximizing the worst-case achievable rate for SUs.

\section{A. Transmission Model}

As shown in Fig. 1, a cognitive radio enabled multi-user downlink cellular network which consists of one primary BS and many primary users (PUs), termed as primary network, is underlaid by a secondary cellular network composed of one secondary BS and several secondary users (SUs). This secondary cellular network is thus an underlaying cognitive NOMA network, which reuses the same spectrum resources as the primary network. There are $M$ SUs in the secondary network, denoted by $\mathcal{M}=\{1, \ldots, M\}$, and $N$ PUs in the primary network, denoted by $\mathcal{N}=\{1, \ldots, N\}$. In the considered cognitive setup, we divide the whole bandwidth for downlink transmission into many equal-length orthogonal subchannels, each with a bandwidth of $B \mathrm{~Hz}$, and at each subchannel NOMA is adopted which allows one subchannel to be shared by multiple SUs simultaneously. The whole orthogonal subchannel set is denoted by $\mathcal{K}^{v}=\left\{1, \ldots, K^{v}\right\}$, and $S_{k}, k \in \mathcal{K}^{v}$, denotes the set of active SUs at subchannel $k$. During the secondary downlink transmission, SC is adopted at the secondary BS while SIC is implemented at each of the SUs to decode their respective information. Under the assumption that there are a maximum of $q$ SUs permitted to access one subchannel simultaneously, SU $m$ is capable of cancelling all the interference caused by the other SUs within the same $\mathcal{S}_{k}$ that have smaller normalized ${ }^{1}$ channel gain than itself. For example, at the stronger cell-center SU, the signal of the cell-edge user is first decoded followed by that of the cell-middle SU, and then that of itself. Whereas, at the weaker cell-edge SU, it directly decodes its information treating interference as noise. Additionally, we assume that the cognitive BS is able to sense the set of vacant subchannels, $\mathcal{K}^{v_{0}}=\left\{1,2, \ldots, K^{v_{0}}\right\}$, from the set of unavailable subchannels, $\mathcal{K}^{v_{1}}=\left\{1,2, \ldots, K_{1}^{v}\right\}$, such that $\mathcal{K}^{v_{0}} \cup \mathcal{K}^{v_{1}}=\mathcal{K}^{v}$.

In the above underlaying NOMA networks, the secondary BS and each SU are equipped with one single antenna. We assume that the BS have perfect channel state information (CSI). We also assume that the BS is able to perform a series of spectral sensing as well as resource allocation algorithms and send the system design variables to the SUs over a separate control channel. The allocated power for SU $m \in \mathcal{M}$ over subchannel $k \in \mathcal{K}^{v_{0}}$ is $P_{m}^{k}$, and the total transmitting power is $P^{\text {total }}$ for the secondary BS. We also assume slow fading with channel coefficients following Rayleigh distribution, and thus

\footnotetext{
${ }^{1}$ The channel gain is normalized by the sum of interference from the primary network and the AWGN at the SU's receiver.
} 


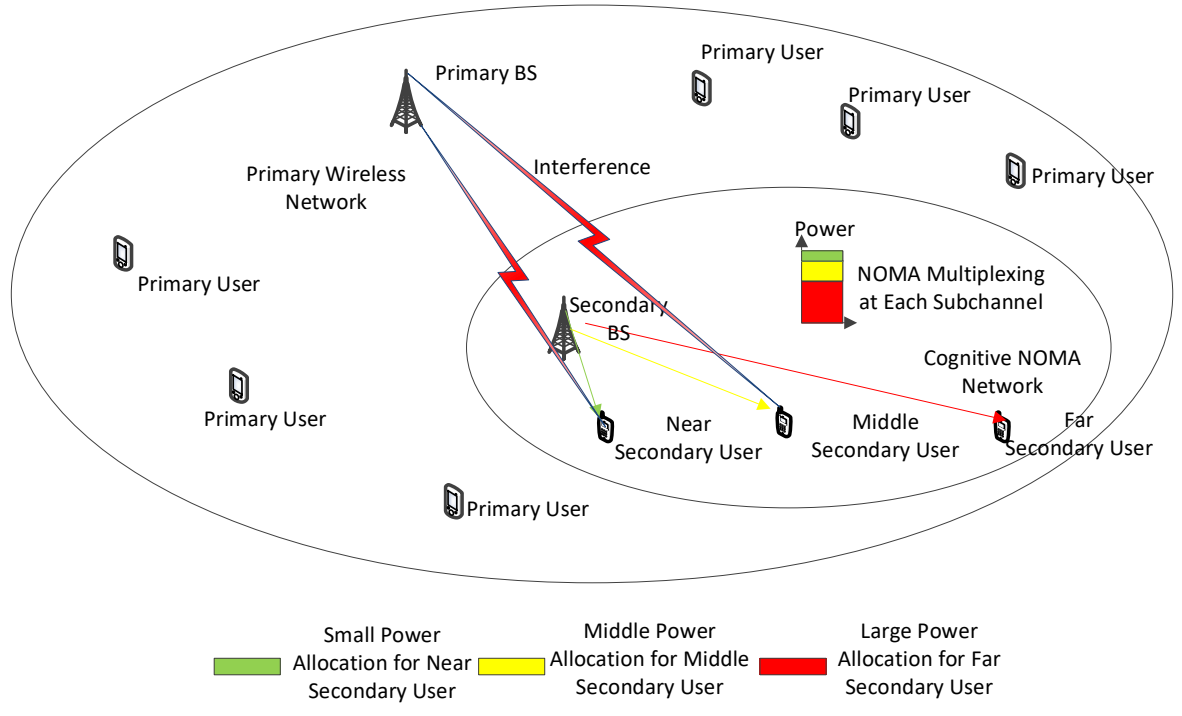

Fig. 1. Underlaying cognitive NOMA networks reusing the same spectrum resources as the primary network.

the CSI remains constant during one transmission interval, and may vary from one interval to another. The channel gain over subchannel $k$ from the secondary BS to the SU $m$ is denoted by $g_{m}^{k}, m \in \mathcal{M}, k \in \mathcal{K}^{v_{0}}$, and the transmission symbol for SU $m$ over subchannel $k$ is denoted by $x_{m}^{k}, m \in \mathcal{M}, k \in \mathcal{K}^{v_{0}}$. The signal that SU $m$ receives over subchannel $k$ is thus expressed as

$$
y_{m}^{k}=g_{m}^{k} \sum_{i \in S_{k}} \sqrt{P_{i}^{k}} x_{i}^{k}+n_{m}^{k},
$$

where $S_{k}$ denots the subset of active SUs over subchannel $k$, $x_{m}^{k}$ is SU $m$ 's transmitted symbol, and $n_{m}^{k}$ is the additive white Gaussian noise (AWGN) for SU $m$ over subchannel $k$. Upon receiving $y_{m}^{k}$, the receiver at $\mathrm{SU} m$ cancels the co-channel interference from any other $S U_{i}, i \in \mathcal{S}_{k}$, with a smaller normalized channel gain by means of SIC. However, since SIC causes considerable hardware complexity with increasing number of multiplexing SUs over the same subchannel, $q$ is defined as the maximum number of users that are allowed to simultaneously access one subchannel to strike balance between the decoding complexity and the multiplexing gain.

\section{B. Interference Temperature Model}

Additionally, we adopt an interference temperature model to characterize the interference caused by the secondary NOMA network to the primary network [1]. At each subchannel $j$, the spectrum spans from $f_{s}+(j-1) B$ to $f_{s}+j B$, where $f_{s}$ is the starting frequency of the first subchannel. When the secondary BS transmits data over subchannel $k$ to SU $m$ with the unit power, there occurs a cross interference to the PU $n$ over subchannel $j$, denoted by $I_{m n}^{k j}$, which is expressed as [22]

$$
I_{m n}^{k j}=\int_{(j-1) B-(k-0.5) B}^{j B-(k-0.5) B} h_{n}^{k} \phi(f) d f,
$$

where $h_{n}^{k}$ is the channel gain from the secondary BS to the PU $n$ over subchannel $k$. The function $\phi(f)$ is the power spectrum density for the multicarrier NOMA signal, and is defined as

$$
\phi(f)=T\left(\frac{\sin \pi f T}{\pi f T}\right)^{2}
$$

where $T$ is the symbol duration.

Hence, the cross-interference to the PU $n$ caused by transmission from the secondary BS to the SU $m$ over the vacant subchannel $k \in \mathcal{K}^{v_{1}}$, denoted by $I_{m n}^{k}$, is given by

$$
I_{m n}^{k}=\sum_{j \in \mathcal{K}^{v_{1}}} I_{m n}^{k j} .
$$

\section{SU's Achievable Rate}

At the physical layer, the achievable rate at which the secondary BS communicates with the SU $m$ over subchannel $k$ is

$$
R_{m}^{k}=B \log _{2}\left(1+\operatorname{SINR}_{m k}\right),
$$

where the signal-to-interference-plus-noise ratio (SINR) for $\mathrm{SU} m$ over subchannel $k$ is given by

$$
\operatorname{SINR}_{m k}=\frac{P_{m}^{k} g_{m}^{k}}{B n_{0}+I_{m k}^{s}},
$$

where $n_{0}$ is the noise power spectral density, and $B$ is the bandwidth for each subchannel.

After employing SIC decoder, the remaining interference power, $I_{m k}^{s}$, introduced by the other SUs to the SU $m$ within the same $\mathcal{S}_{k}$ over subchannel $k$, denoted by $I_{m k}^{s}$, is

$$
I_{m k}^{s}=\sum_{l \in \mathcal{M}^{*}} P_{l}^{k} g_{m}^{k},
$$

where $\mathcal{M}^{*}$ denotes the set of SUs, whose channel power gain are larger than that of SU $m$ over subchannel $k(k \in \mathcal{K})$, i.e., $\mathcal{M}^{*}=\left\{m^{*} \in \mathcal{M} \mid g_{m^{*}}^{k}>g_{m}^{k}\right\}$. Since SU $m$ decodes the information transmitted by the secondary BS over all 
the subchannels in $\mathcal{K}^{v_{0}}$ via orthogonal-frequency division multiplexing (OFDM), the achievable transmission rate for SU $m$ is given by

$$
R_{m}=\sum_{k \in \mathcal{K}^{v_{0}}} \alpha_{m}^{k} R_{m}^{k}
$$

where $\alpha_{m}^{k}$ 's is an NOMA-OFDMA subchannel assignment variable indicating whether subchannel $k$ is assigned to $\mathrm{SU}$ $m$ or not, which is given by

$$
\alpha_{m}^{k}=\left\{\begin{array}{l}
1, \text { if subchannel } k \text { is allocated to } \mathrm{SU} m, \\
0, \text { otherwise. }
\end{array}\right.
$$

\section{Problem Formulation}

The power consumption consists of two components at the secondary BS [23]: the first component is $P_{c}$, which is a fixed consumption power by the circular system; and the second component is the dynamic downlink-transmission power. Therefore, the total consumption power at the secondary BS is given by $P_{B S}=P_{c}+\sum_{m \in \mathcal{M}} \sum_{k \in \mathcal{K}^{v_{0}}} \alpha_{m}^{k} P_{m}^{k}$. The total power consumption, $P_{B S}$, must satisfy the maximum power constraint, i.e., $P_{B S} \leq P^{\text {total }}$, which is given by

$$
P_{c}+\sum_{m \in \mathcal{M}} \sum_{k \in \mathcal{K}^{v} 0} \alpha_{m}^{k} P_{m}^{k} \leq P^{\text {total }} .
$$

In this work, 'power allocation' refers to optimization of the downlink transmitting power of the secondary BS for a particular user $m, m \in \mathcal{M}$, over a particular subcarrier $k$, $k \in \mathcal{K}^{v_{0}}$, denoted by $P_{m}^{k}$, (cf. (6) in the paper), while 'power consumption' means the total amount of power consumed by, e.g., the secondary BS, which includes the static power consumed by the hardware circuit and dynamic downlink transmitting power specified by the power allocation algorithm (cf. (10)).

Moreover, the maximum number of SUs that one subchannel can accommodate leads to the following constraint:

$$
\sum_{m \in \mathcal{M}} \alpha_{m}^{k} \leq q, \forall k \in \mathcal{K}^{v_{0}},
$$

which is an integer constraint, since all $\alpha_{m}^{k}$ are integer variables. In addition, the total cross-interference to the primary network should satisfy the interference temperature threshold set for PU $n$, i.e.,

$$
\sum_{m \in \mathcal{M}} \sum_{k \in \mathcal{K}^{v_{0}}} \alpha_{m}^{k} P_{m}^{k} I_{m n}^{k} \leq I_{n}^{\mathrm{th}}, \forall n \in \mathcal{N},
$$

where $I_{n}^{\text {th }}$ 's is the maximum amount of cross-interference that PU $n, n \in \mathcal{N}$ can tolerate [24].

To advocate fairness in terms of the achievable downlink transmission rate among different SUs, we aim for maximizing the minimum downlink transmission rate among the SUs subject to the total transmitting power constraint (10) at the secondary BS, the maximum number of multiplexing SUs per subchannel (c.f. (11)), and the interference temperature constraints imposed by the co-existing PUs (c.f. (12)). Consequently, the joint NOMA-SU assignment and power allocation problem is formulated as below:

$$
\begin{aligned}
\text { (OP1) : } & \max _{\left\{\alpha_{m}^{k}\right\},\left\{P_{m}^{k}\right\}}\left\{\min _{m \in \mathcal{M}} R_{m}\right\} \\
& \text { Subject to } \\
& (10)-(12), \\
& \alpha_{m}^{k} \in\{0,1\}, P_{m}^{k} \geq 0, \forall m \in \mathcal{M}, \forall k \in \mathcal{K}^{v_{0}} .
\end{aligned}
$$

\section{NOMA-SU ASSIGNMENT AND POWER Allocation}

\section{ALGORITHM}

The problem (OP1) is a mixed-integer nonlinear programming (MINLP), and thus difficult to solve directly. In addition, since $\alpha_{m}^{k}$ 's and $P_{m}^{k}$ 's are given, this problem is still non-convex due to the occurrence of $P_{m}^{k}$ 's in both nominator and denominator of SINR $m k$ 's (c.f. (6)). To make its solution tractable, we decouple the original problem into a NOMA-SU assignment problem and a power allocation problem. Specifically, with fixed power allocation, we propose a heuristic NOMA-SU assignment algorithm. The heuristic algorithm is designed based on the fairness criterion and the constraints of the interference power and power multiplexing. Then, with fixed NOMA-SU assignment policy, successive convex approximation (SCA) is adopted to obtain sub-optimal power allocation for the power allocation problem. Then, we alternatively solve the above two problems to jointly optimize the NOMA-SU assignment and the power allocation for problem (OP1). Finally, the complexity of the algorithm is analyzed.

\section{A. NOMA-SU assignment}

With the power allocation in (OP1) fixed as $\bar{P}_{m}^{k}, m \in \mathcal{M}$, $k \in \mathcal{K}$, the NOMA-SU assignment problem that determines the worst-case achievable rate for the SUs is denoted by (OP2), i.e.,

$$
\begin{aligned}
(\mathrm{OP} 2): & \max _{\left\{\alpha_{m}^{k}\right\}} \min _{m \in \mathcal{M}} R_{m} \\
& \text { Subject to } \\
& (10)-(12), \alpha_{m}^{k} \in\{0,1\}, \forall m \in \mathcal{M}, \forall k \in \mathcal{K}^{v_{0}} .
\end{aligned}
$$

In order to solve problem (OP2), a heuristic NOMA-SU assignment algorithm is proposed in Algorithm 1, which is implemented by the secondary BS. In Algorithm 1, step 1 initializes the power allocation, SU assignment, and transmission rate for each SU. Step 2- step 12 perform the SU assignment for each subchannel. Assuming that the vacant subchannels $\mathcal{K}^{v_{0}}$ having been sensed a priori by the secondary BS, first, we allocate the total power equally among all SUs over different subchannels in step 1. In step 2- step 12, for each subchannel, the secondary BS selects the SU with the minimum achievable rate over all the subchannels in $\mathcal{K}_{0}^{v}$, namely, $R_{m}$ (c.f. (8)). If the interference caused to each of the PUs does not exceed the interference temperature constraints, and the current subchannel can accommodate more SUs, assign the selected SU to the current subchannel; otherwise, proceed with the next subchannel until the interference constraint is not met any more (cf. step 4 in Algorithm 1). Note that step 3 of Algorithm 1 , if multiple secondary users have the same transmission rate, we select the SU with the largest channel power gain over the current subchannel. 


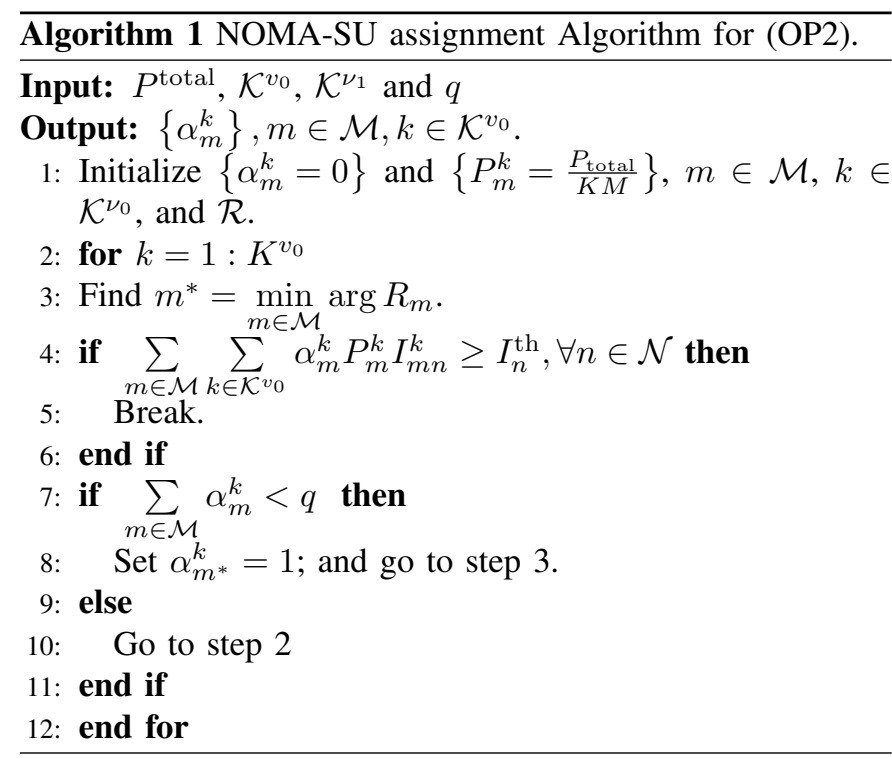

\section{B. Power Allocation for the Secondary BS}

In the sequel, we allocate the power among all SUs over the selected subchannels to improve the worst-case fairness in terms of the achievable transmission rate of the SUs by solving the following problem:

$$
\begin{aligned}
(\mathrm{OP} 3): & \max _{\left\{P_{m}^{k}\right\}}\left\{\min R_{m}\right\} \\
& \text { Subject to } \\
& (10),(12), P_{m}^{k} \geq 0, \forall m \in \mathcal{M}, \forall k \in \mathcal{K}^{v_{0}} .
\end{aligned}
$$

The problem (OP3) can be recast into its equivalent epigraph form:

$$
\begin{aligned}
\left(\mathrm{OP}^{\prime}\right): & \max _{\left\{P_{m}^{k}\right\}, \psi} \psi \\
& \text { Subject to } \\
& R_{m} \geq \psi, \forall m \in \mathcal{M}, \\
& (10),(12), \\
& P_{m}^{k} \geq 0, \forall m \in \mathcal{M}, \forall k \in \mathcal{K}, \psi \geq 0 .
\end{aligned}
$$

where $\psi$ is an auxiliary variable. However, since problem $\left(\mathrm{OP} 3^{\prime}\right)$ is a non-convex optimization problem due to the fractional forms of $R_{m}$ 's (c.f. (6)) in (16a), the SCA method is utilized to obtain a lower bound solution for $\left(\mathrm{OP} 3^{\prime}\right)$ [25].

Proposition 1: The power allocation problem (OP4) is a convex resource allocation problem.

Proof: See Proof of Proposition 1.

In the $(j+1)$ th iteration of the SCA method, we aim for solving the following problem:

$$
\begin{aligned}
(\text { OP } 4): & \max _{\left\{\hat{P}_{m}^{k}\right\}, \psi} \psi \\
& \text { Subject to } \\
& P_{c}+\sum_{m \in \mathcal{M}} \sum_{k \in \mathcal{K}^{v_{0}}} \alpha_{m}^{k} \exp \left(\hat{P}_{m}^{k}\right) \leq P^{\text {total }}, \quad \text { (17a) } \\
& \sum_{k \in \mathcal{K}^{v_{0}}} \chi_{m k}-\psi \geq 0, \forall m \in \mathcal{M}, \\
& \sum_{m \in \mathcal{M}} \sum_{k \in \mathcal{K}^{v_{0}}} \alpha_{m}^{k} I_{m n}^{k} \exp \left(\hat{P}_{m}^{k}\right) \leq I_{n}^{t h}, \forall n \in \mathcal{N},
\end{aligned}
$$

and

$$
\begin{aligned}
& \chi_{m k}= \\
& \alpha_{m}^{k} B\left[b_{m k} \log _{2}\left(\exp \left(\hat{P}_{m}^{k}\right) g_{m}^{k}\right)-B_{m k}\left(\left\{\hat{P}_{l}^{k}\right\}_{l \in \mathcal{M}^{*}}\right)\right]
\end{aligned}
$$

where $B_{m k}\left(\left\{\hat{P}_{l}^{k}\right\}_{l \in \mathcal{M}^{*}}\right), \forall m \in \mathcal{M}, \forall k \in \mathcal{K}^{v_{0}}$ is defined as

$$
\begin{aligned}
& B_{m k}^{\{j\}}\left(\left\{\hat{P}_{l}^{k}\right\}_{l \in \mathcal{M}^{*}}\right)= \\
& b_{m k}^{\{j\}} \log _{2}\left(B n_{0}+\sum_{l \in \mathcal{M}^{*}} \exp \left(\hat{P}_{l}^{k}\right) g_{m}^{k}\right)-c_{m k}^{\{j\}}
\end{aligned}
$$

where the constraints (17a), (17b) and (17c) are obtained by substituting $\exp \left(\hat{P}_{m}^{k}\right)$ 's for $P_{m}^{k}$ 's in (10), (16a) and (12), respectively.

Now that $B_{m k}\left(\left\{\hat{P}_{l}^{k}\right\}_{l \in \mathcal{M}}\right)$ is a convex function w.r.t. $\hat{P}_{l}^{k}$, $l \in \mathcal{M}^{*}$ [page 119,24], together with the other convex constraints (c.f. (17a) and (17c)) that are easily verified, problem (OP4) turns out to be a convex problem. Hence, we solve it by Lagrangian duality method in the sequel. First, the Lagrangian of problem (OP4) is

$$
\begin{aligned}
& f\left(v, u_{m}, \delta_{n}, \hat{P}_{m}^{k}, \psi\right)=\left(1-\sum_{m \in \mathcal{M}} u_{m}\right) \psi \\
& +v\left\{P^{\text {total }}-\left(P_{c}+\sum_{m \in \mathcal{M}} \sum_{k \in \mathcal{K}^{v_{0}}} \alpha_{m}^{k} \exp \left(\hat{P}_{m}^{k}\right)\right)\right\} \\
& +\sum_{n \in \mathcal{N}} \delta_{n}\left\{I_{n}^{t h}-\sum_{m \in \mathcal{M}} \sum_{k \in \mathcal{K}^{v_{0}}} \alpha_{m}^{k} I_{m n}^{k} \exp \left(\hat{P}_{m}^{k}\right)\right\} \\
& +\sum_{m \in \mathcal{M}} \sum_{k \in \mathcal{K}^{v_{0}}}\left(1+u_{m}\right) \chi_{m k},
\end{aligned}
$$

where $v, u_{m}$ 's, and $\delta_{n}$ 's are the Lagrangian multipliers corresponding to (17a), (17b) and (17c), respectively. In line with the Lagrangian given by $(20), h\left(v, u_{m}, \delta_{n}\right)$ is the dual function and can be expressed as

$$
h\left(v, u_{m}, \delta_{n}\right)=\max _{\hat{P}_{m}^{k} \geq 0, \psi \geq 0} f\left(v, u_{m}, \delta_{n}, \hat{P}_{m}^{k}, \psi\right)
$$

The dual problem of (OP4) is thus given by

$$
\begin{aligned}
(\mathrm{OP} 4-\text { dual }): & \min _{v,\left\{u_{m}\right\},\left\{\delta_{n}\right\}} h\left(v, u_{m}, \delta_{n}\right) \\
& \text { Subject to } \\
& v \geq 0, u_{m} \geq 0, \delta_{n} \geq 0, \forall m \in \mathcal{M}, \forall n \in \mathcal{N} .
\end{aligned}
$$

Next, to calculate the dual function in (22), we resort to the following lemma.

Lemma 1: With any fixed dual variables $v, u_{m}$ 's, and $\delta_{n}$ 's, the optimal power allocation $\hat{P}_{m}^{k}, m \in M, k \in K$, and $\psi$ are 
given by

$$
\hat{P}_{m}^{k}=\left[\ln \left(\frac{B b_{m k}\left(1+u_{m}\right) / \ln 2}{\left(v+\sum_{n \in \mathcal{N}} \delta_{n} I_{m n}^{k}\right)}\right)\right]^{+}, \forall m \in \mathcal{M}, k \in \mathcal{K}^{v_{0}} .
$$

\section{Proof: See Proof of Lemma 1.}

Finally, the optimum values of $v, u_{m}$ 's, and $\delta_{n}$ 's for the dual problem can be calculated by sub-gradient descent method, i.e.,

$$
\begin{aligned}
& \left\{\begin{array}{l}
v^{(i+1)}=\left[v^{(i)}-\Delta \varepsilon_{1} P_{v}^{(i+1)}\right]^{+}, \\
P_{v}^{(i+1)}=P^{\text {total }}-P_{c}-\sum_{m \in \mathcal{M}} \sum_{k \in \mathcal{K}^{v_{0}}} \alpha_{m}^{k} \exp \left(\hat{P}_{m}^{k}\right),
\end{array}\right. \\
& \left\{\begin{array}{l}
\delta_{n}^{(i+1)}=\left[\delta_{n}^{(i)}-\Delta \varepsilon_{2} I_{\delta}^{(i+1)}\right]^{+}, \\
I_{\delta}^{(i+1)}=I_{n}^{t h}-\sum_{m \in \mathcal{M}} \sum_{k \in \mathcal{K}^{v_{0}}} \alpha_{m}^{k} I_{m n}^{k} \exp \left(\hat{P}_{m}^{k}\right),
\end{array}\right.
\end{aligned}
$$

and

$$
u_{m}^{(i+1)}=\left[u_{m}^{(i)}-\Delta \varepsilon_{3}\left(\sum_{k \in \mathcal{K}^{v_{0}}} \chi_{m k}-\psi\right)\right]^{+},
$$

where $\Delta \varepsilon_{1}, \Delta \varepsilon_{2}$ and $\Delta \varepsilon_{3}$ are small step sizes, and $i$ is the iteration index. Since the Lipchitz continuity condition is satisfied for the gradient of problem (OP4-dual), we can guarantee the optimum solution with convergence by iteratively updating $\nu, \delta_{n}$ 's, and $u_{m}$ 's, using (24)-(26) with appropriate step sizes [26]. In addition, $v^{(i)}, u_{m}^{(i)}$ 's, and $\delta_{n}^{(i)}$ 's denote the values for $v, u_{m}$ 's, and $\delta_{n}$ 's at the $(i)$ th iteration.

To sum up, the proposed SCA-based power allocation algorithm for solving (OP3') is summarized in Algorithm 2. In Algorithm 2, $b_{m k}^{(j)}$,s and $c_{m k}^{(j)}$ 's are required to be updated by $\overline{\operatorname{SINR}}_{m k}^{(j)}$ 's using the solution $\hat{P}_{m}^{k(j)}$, s for (OP4) obtained in the $j$ th iteration, where $\epsilon_{4}$ is the maximum tolerance, $I_{\max }$ is the maximum iterative number, and $i$ and $j$ are the iteration indexes. $P_{m}^{k(j)}$ 's, $\hat{P}_{m}^{k(j)}$ 's, $b_{m k}^{(j)}$ 's, $c_{m k}^{(j)}$ 's, $\overline{\operatorname{SINR}}_{m k}^{(j)}$ 's, $\left\{I_{m k}^{s(j)}\right\}$ 's, $B_{m k}^{(j)}$ 's and $\varphi^{(j)}$ are the values for $P_{m}^{k}$ 's, $\hat{P}_{m}^{k}$ 's, $b_{m k}$ 's, $c_{m k}$ 's, $\overline{\text { SINR }}_{m k}$ 's, $\left\{I_{m k}^{s}\right\}$ 's, $B_{m k}$ 's and $\varphi$ at the $j$ th iteration. In addition, $v^{(i)}, u_{m}^{(i)}$ 's, $\delta_{n}^{(i)}$ s, $\left(\hat{P}_{m}^{k}\right)^{(i)}$ 's denote the values for $v, u_{m}$ 's, $\delta_{n}$ 's, $\hat{P}_{m}^{k}$ 's at the $i$ th iteration; $\psi^{*}, v^{*}, u_{m}^{*}$ 's, $\delta_{n}^{*}$ 's, $\left(P_{m}^{k}\right)^{*}$ 's and $\left(\hat{P}_{m}^{k}\right)^{*}$ 's are the values for $\psi, v, u_{m}$ 's, $\delta_{n}$ 's, $P_{m}^{k}$ 's and $\hat{P}_{m}^{k}$ 's at the termination iteration; and $\psi_{(i)}^{(j)}$ is the value for $\psi$ in the $i$ th inner iteration (the subgradient method) of the $j$ th outer iteration (the SCA method). Algorithm 2 converges to a KKT solution according to Theorem 1 in [27]. Note that, since problem $\left(\mathrm{OP} 3^{\prime}\right)$ is tightened by solving a series of convex optimization problem given by problem (OP4), solution achieved by Algorithm 2 serves as a lowerbound for the problem $\left(\mathrm{OP}^{\prime}\right)$ in general [28]-[30].

$\overline{\text { Algorithm } 2 \text { Power Allocation for }\left(\mathrm{OP} 3^{\prime}\right) .}$
Input: $P^{\text {total }}, I_{\max },\left\{\alpha_{m}^{k}\right\},\left\{I_{m n}^{k}\right\}$ and $\left\{I_{n}^{\text {th }}\right\}, m \in \mathcal{M}, k \in$
$\mathcal{K}^{v_{0}}, n \in \mathcal{N}$.

Output: $\left\{P_{m}^{k}\right\}$ and $\psi, m \in \mathcal{M}, k \in \mathcal{K}^{v_{0}}$.

1: Initialize $j=0,\left\{\overline{\operatorname{SINR}}_{m k}^{(j)}=0\right\},\left\{I_{m k}^{s(j)}=0\right\}$, and $\psi^{(j)}=0, m \in \mathcal{M}, k \in \mathcal{K}^{v_{0}}$.

2: Calculate $\left\{b_{m k}^{(j)}\right\},\left\{c_{m k}^{(j)}\right\}$ and $\left\{B_{m k}^{(j)}\right\}$ using $\overline{\operatorname{SINR}}_{m k}^{(j)}$ in accordance with (33), (34) and (19).

3: repeat

4: $\quad j:=j+1$

5: $\quad$ Initialize $i=0, v^{(i)},\left\{u_{m}^{(i)}\right\}$, and $\left\{\delta_{n}^{(i)}\right\}, m \in \mathcal{M}$, $n \in \mathcal{N}$

6: $\quad$ repeat

7: $\quad$ Calculate $\left(\hat{P}_{m}^{k}\right)^{(i)} m \in \mathcal{M}, k \in \mathcal{K}^{v_{0}}$, as follows

$$
\left(\hat{P}_{m}^{k}\right)^{(i)}=\left[\ln \left(\frac{B b_{m k}^{(j)}\left(1+u_{m}^{(i)}\right) / \ln 2}{\left(v^{(i)}+\sum_{n \in \mathcal{N}} \delta_{n}^{(i)} I_{m n}^{k}\right)}\right)\right]^{+} .
$$

8: $\quad$ Calculate $\psi_{(i)}^{(j)}$ as follows

$$
\psi_{(i)}^{(j)}=\left\{\begin{array}{l}
+\infty, 1-\sum_{m \in \mathcal{M}} u_{m}^{(i)} \geq 0 . \\
0, \text { otherwise }
\end{array}\right.
$$

9:

10 :

11:

12:

13:

14:

$15:$

16 :

$17:$

18:

19 :

20:

20:

21: if $i<I_{\max }$ then

$i:=i+1$.

Update $v^{(i)},\left\{u_{m}^{(i)}\right\}$, and $\left\{\delta_{n}^{(i)}\right\}$ in accordance with (24)-(26).

else

Update $v^{*}=v^{(i)},\left\{u_{m}^{*}=u_{m}^{(i)}\right\}$, and $\left\{\delta_{n}^{*}=\delta_{n}^{(i)}\right\}$.

Obtain $\left(\hat{P}_{m}^{k}\right)^{*}$ as

$$
\left(\hat{P}_{m}^{k}\right)^{*}=\left[\ln \left(\frac{B b_{m k}^{(j)}\left(1+u_{m}^{*}\right) / \ln 2}{\left(v^{*}+\sum_{n \in \mathcal{N}} \delta_{n}^{*} I_{m n}^{k}\right)}\right)\right]^{+}
$$

and

$$
\left(P_{m}^{k}\right)^{*}=\exp \left(\left(\hat{P}_{m}^{k}\right)^{*}\right)
$$

Obtain $\psi^{*}$ as

$$
\left\{\begin{array}{l}
\psi^{*}=\min _{m \in \mathcal{M}} \sum_{k \in \mathcal{K}^{v_{0}}} \chi_{m k}^{(j)} \\
\chi_{m k}^{(j)}=\alpha_{m}^{k} B\left[b_{m k}^{(j)} \log _{2}\left(\left(P_{m}^{k}\right)^{*} g_{m}^{k}\right)-B_{m k}^{(j)}\right] .
\end{array}\right.
$$

end if

until $i=I_{\max }$

Update $\left\{P_{m}^{k(j)}=\left(P_{m}^{k}\right)^{*}\right\}$ and $\psi^{(j)}=\psi^{*}$.

Update $\left\{I_{m k}^{s(j)}\right\}$ and $\left\{\overline{\operatorname{SINR}}_{m k}^{(j)}\right\}$ using (6) and (7).

Update $\left\{b_{m k}^{(j)}\right\},\left\{c_{m k}^{(j)}\right\}$ and $\left\{B_{m k}^{(j)}\right\}$ using $\overline{\operatorname{SINR}}_{m k}^{(j)}$ in accordance with (33), (34) and (19).

until $\left|\psi^{(j)}-\psi^{(j-1)}\right| \leq \epsilon_{4}$

Obtain $\left\{P_{m}^{k}=P_{m}^{k(j)}\right\}$ and $\psi=\psi^{(j)}$. 


\section{Iterative Power Allocation and NOMA-SU assignment}

To solve problem (OP1), an iterative algorithm is proposed to jointly optimize the NOMA-SU assignment and power allocation for the underlaying NOMA networks. Specifically, at the initial stage, $\left\{P_{m}^{k(0)}\right\}$ 's is initialized by allocating equal power to $M$ SUs over $K$ subchannels. Then at the $t$ th iteration, $t=1,2, \ldots,\left\{\alpha_{m}^{k(t)}\right\}$ 's is obtained by Algorithm 1 with the power allocation fixed by $\left\{P_{m}^{k(t-1)}\right\}$ 's and $\left\{P_{m}^{k(t)}\right\}$ 's is updated by solving problem $\left(\mathrm{OP}^{\prime}\right)$ using the SCA-based algorithm proposed in Algorithm 2 with the NOMA-SU assignment fixed by $\left\{\alpha_{m}^{k(t)}\right\}$ 's. In Algorithm 2, step 1 initializes the variables $\overline{\operatorname{SINR}}_{m k}^{(j)}$ and $I_{m k}^{s(j)}$. Step 2 initializes the variables $\left\{b_{m k}^{(j)}\right\},\left\{c_{m k}^{(j)}\right\}$ and $\left\{B_{m k}^{(j)}\right\}$. Step 3 and step 4 update the iterative variable $j, i, v^{(i)},\left\{u_{m}^{(i)}\right\}$, and $\left\{\delta_{n}^{(i)}\right\}$. Step 6-step 16 calculate the power allocation and step 9-step 15 calculate the Lagrangian multipliers $v^{(i)}$, $\left\{u_{m}^{(i)}\right\}$, and $\left\{\delta_{n}^{(i)}\right\}$. Step 17- step 19 update the other variables $\left\{P_{m}^{k(j)}=\left(P_{m}^{k}\right)^{*}\right\},\left\{P_{m}^{k(j)}=\left(P_{m}^{k}\right)^{*}\right\},\left\{\overline{\operatorname{SINR}}_{m k}^{(j)}\right\},\left\{b_{m k}^{(j)}\right\}$, $\left\{c_{m k}^{(j)}\right\}$, and $\left\{B_{m k}^{(j)}\right\}$ using $\overline{\operatorname{SINR}}_{m k}^{(j)}$ at the $j$ th iteration. Step 21 outputs the power allocation result.

The above alternating procedure is repeated until no further improvement is made [25], [31], which is summarized in Algorithm 3, where $\epsilon$ denotes a tolerance controlling the accuracy of Algorithm 3. In Algorithm 3, step 1 initializes the variables. Step 3 calculates the SU assignment variable $\left\{\hat{\alpha}_{m}^{k(t)}\right\}$ with the fixed power allocation $\left\{P_{m}^{k(t-1)}\right\}$. Step 4 calculates the power allocation $\left\{\hat{P}_{m}^{k(t)}\right\}$ with the fixed $\left\{\hat{\alpha}_{m}^{k(t)}\right\}$. Step 5step 7 update the latest power allocation and SU assignment. $f\left(\left\{P_{m}^{k}, \alpha_{m}^{k}\right\}\right)$ denotes the objective function of (OP1), which is obtained by plugging the corresponding $\left\{P_{m}^{k}, \alpha_{m}^{k}\right\}$ into (5). $\left\{R_{m}^{(t)}\right\}$ 's and $\left\{R_{m}^{(t-1)}\right\}$ 's are the values for $R_{m}$ at the $t$ th iteration and at the $(t-1)$ th iteration, respectively.

\section{Algorithm Complexity Analysis}

The algorithm complexity of Algorithm 1 for the heuristic NOMA-SU assignment is $O\left(3 M K^{v_{0}} q\right)$. The algorith$\mathrm{m}$ complexity of Algorithm 2 for the power allocation is given by $O\left(O_{I} I_{\max } M^{2} q K^{v_{0}}\right)$, where $I_{\max }$ is the maximum iterative number for step 3 - step 6 , and $O_{I}$ is the iterative number for step 2- step 9 (c.f. Algorithm 2). Hence, the overall algorithm complexity for Algorithm 3 is $O\left(\left(3 M K^{v_{0}} q+O_{I} I_{\max } M^{2} q K^{v_{0}}\right) O_{s p}\right)$, where $O_{s p}$ is the number of iteration controlled by $\varepsilon$ in Algorithm 3. The computational complexity analysis for the proposed algorithm is in an average sense.

\section{NumERICAL EXAMPLES}

\section{A. Simulation Setup}

In this section, the performance of NOMA-SU assignment and power allocation is evaluated for the underlaying NOMA $\overline{\text { Algorithm } 3 \text { Iterative Joint NOMA-SU assignment and Power }}$ Allocation.

Input: $\epsilon, P^{\text {total }}, \mathcal{K}^{v_{0}}, \mathcal{K}^{\nu_{1}}, q, I_{\max },\left\{\alpha_{m}^{k}\right\},\left\{I_{m n}^{k}\right\}$ and $\left\{I_{n}^{\text {th }}\right\}, m \in \mathcal{M}, k \in \mathcal{K}^{v_{0}}, n \in \mathcal{N}$.

Output: $\left\{\alpha_{m}^{k(t)}\right\}$ and $\left\{P_{m}^{k(t)}\right\}, m \in \mathcal{M}, \forall k \in \mathcal{K}^{v_{0}}$.

1: Initialize $t=1$ and $\left\{P_{m}^{k(0)}\right\}$ with equal power allocation.

2: repeat

3: $\quad$ For a fixed $\left\{P_{m}^{k(t-1)}\right\}$, find NOMA-SU assignment $\left\{\hat{\alpha}_{m}^{k(t)}\right\}$ by solving the problem (OP2) using Algorithm 1.

4: For a fixed $\left\{\hat{\alpha}_{m}^{k(t)}\right\}$, find power allocation $\left\{\hat{P}_{m}^{k(t)}\right\}$ by solving the problem $\left(\mathrm{OP} 3^{\prime}\right)$ with Algorithm 2.

\begin{tabular}{ll} 
5: & Set $\quad\left\{P_{m}^{k(t)}, \alpha_{m}^{k(t)}\right\}$ \\
& $\arg \max \left\{f\left(\left\{P_{m}^{k(t-1)}, \hat{\alpha}_{m}^{k(t)}\right\}\right), f\left(\left\{\hat{P}_{m}^{k(t)}, \hat{\alpha}_{m}^{k(t)}\right\}\right)\right\}$. \\
6: & Set $g^{(t)}=\max \left\{f\left(\left\{P_{m}^{k(t-1)}, \hat{\alpha}_{m}^{k(t)}\right\}\right), f\left(\left\{\hat{P}_{m}^{k(t)}, \hat{\alpha}_{m}^{k(t)}\right\}\right)\right\}$. \\
7: & Update $t:=t+1$. \\
8: & until $\left|g^{(t)}-g^{(t-1)}\right| / g^{(t-1)}<\epsilon$ or $g^{(t)}-g^{(t-1)}<0$ \\
\hline
\end{tabular}

TABLE I

SiMULATION PARAMETERS.

\begin{tabular}{ll}
\hline Simulation Parameter & Value \\
\hline$B$ & $1 \mathrm{MHz}$ \\
$P_{c}$ & $20 \mathrm{dBm}$ \\
$n_{0}$ & $1 \times 10^{-19} \mathrm{~W} / \mathrm{Hz}$ \\
$I_{m k}^{p}$ & Gaussian distribution with variance \\
& $1 \times 10^{-15} \mathrm{~W}$ and zero mean \\
Path Loss Fact & 4 \\
\hline
\end{tabular}

networks by numerical examples. The simulation tool is Matlab 2016, and we use the Montle Carlo method to simulate the network operation. There exist one primary network and one underlaying NOMA network. In the primary network, there are two PUs located at distance of $300 \mathrm{~m}$ and $600 \mathrm{~m}$ away from the secondary BS, respectively. In the underlaying NOMA network, the secondary BS is centred with $M$ SUs' distance to the secondary BS uniformly generated from $\mathcal{U}(0,400] \mathrm{m}$. For each subchannel, the probability of PU activity is uniform distributed at the interval $[0,1]$. The other simulation parameters are given in Table I. For the proposed algorithm, there exit two cases, i.e., $q=3$ for NOMA scheme and $q=2$ for NOMA scheme. In [32], Mitran's algorithm focuses on the fairness resource allocation for cognitive OFDMA networks, and we compare Mitran's algorithm to our proposed algorithm to checkout our proposed new scheme. The computational complexity is compared with Mitran's algorithm [32] in table II. In table II, $O_{s}$ is the iterative number for subchannel allocation, and $O_{p}$ is the iterative number for power allocation.

\section{B. Evaluation Metrics}

The throughput and Jain Fairness Index are calculated with the Montle Carlo method. The total throughput of the underlying NOMA networks refers to the sum of throughput of all SUs, which is defined by Eq. (32). The average throughput 
TABLE II

COMPUTATIONAL COMPLEXITY COMPARISON.

\begin{tabular}{ll}
\hline Algorithm Name & Computational Complexity \\
\hline Proposed Algorithm & $O\left(\left(3 M K^{v_{0}} q+O_{I} I_{\max } M^{2} q K^{v_{0}}\right) O_{s p}\right)$ \\
Mitran's Algorithm & $O\left(\left(1+O_{p}+O_{s} M\right)\left(K^{v_{0}}\right)^{3}\right)$ \\
\hline
\end{tabular}

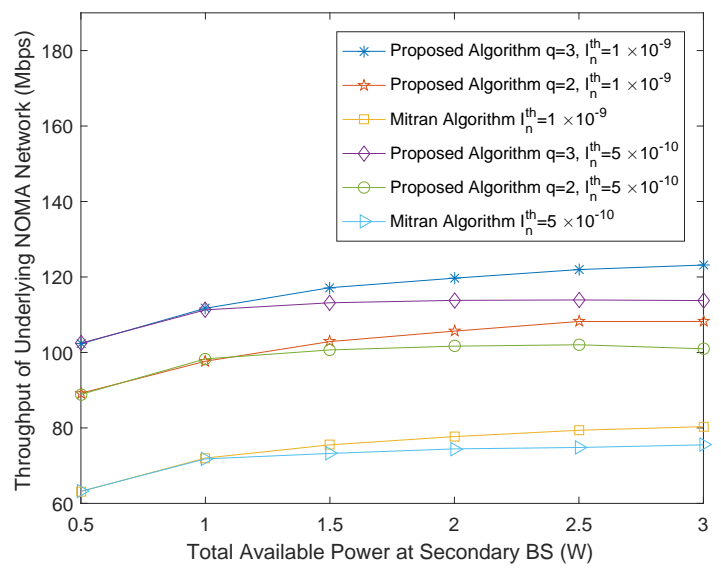

refers to the total throughput divided by the number of SUs, which is defined by Eq. (33). Jain Fairness Index refers to the fairness of a set of values where there are $M$ SUs and $R_{m}$ is the throughput for the $m$ th SU. It ranges from $1 / M$ to $1.1 / M$ is the worst case, 1 is the best case, and it is maximum when all SUs receive the same rate. Jain Fairness Index is defined as Eq. (34). In Eq. (32)-(34), $R_{m}$ is the transmission rate for SU $m, M$ is the total number of SUs in underlying cognitive NOMA networks and $\mathcal{M}$ is the set of SUs.

$$
\begin{gathered}
\text { Throughput }=\sum_{m \in \mathcal{M}} R_{m} \\
\text { Average Throughput }=\frac{\sum_{m \in \mathcal{M}} R_{m}}{M} \\
\text { Jain Fairness Index }=\frac{\left(\sum_{m \in \mathcal{M}} R_{m}\right)^{2}}{M\left(\sum_{m \in \mathcal{M}} R_{m}^{2}\right)}
\end{gathered}
$$

\section{Results Discussion}

We depict the total throughput versus the total power at secondary BS for the underlaying NOMA network in Fig. 2. The simulation parameters are $M=5$ and $K^{v}=16$. There are two cases, i.e., $I_{n}^{\text {th }}=5 \times 10^{-10} \mathrm{~W}$ and $I_{n}^{\text {th }}=1 \times 10^{-9}$ W. In Fig. 2, it can be seen that the total throughput of the underlaying NOMA network increases with the growth of the total power. When the available power for secondary BS grows, secondary BS allocates more power to the SUs to increase the network throughput. However, the growing trend slows down and get saturated when the total power constraint becomes approximately larger than $2.5 \mathrm{~W}$. This is because when the total power of the secondary BS gets larger, the co-channel interference caused to the SU (cf. (7)) increases as well. Thus, to improve the worst SU' throughput, sufficient power has to be allocated to these interfering channels to mitigate the interference power (cf. (6)), which in turn limits the increase in the throughout of all other users and thus the total throughput of the secondary network. We can also see that the network throughput of the proposed algorithm is much larger than that of Mitran algorithm. This is because Mitran algorithm does not take full advantage of the power domain diversity.

We depict the total throughput versus the interference temperature threshold imposed by the PUs in Fig. 3. The simulation parameters are $M=5$ and $K^{v}=16$. There are two cases, i.e., $P^{\text {total }}=0.5 \mathrm{~W}$ and $P^{\text {total }}=1 \mathrm{~W}$. In Fig. 3, it can be observed that the total throughput of the underlaying NOMA network grows with the interference temperature threshold at PU. The throughput of the underlaying NOMA network with $P^{\text {total }}=0.5 \mathrm{~W}$ remains unchanged when the interference temperature threshold at PU exceeds

Fig. 2. Total throughput vs. total power at secondary BS for the underlaying NOMA networks.

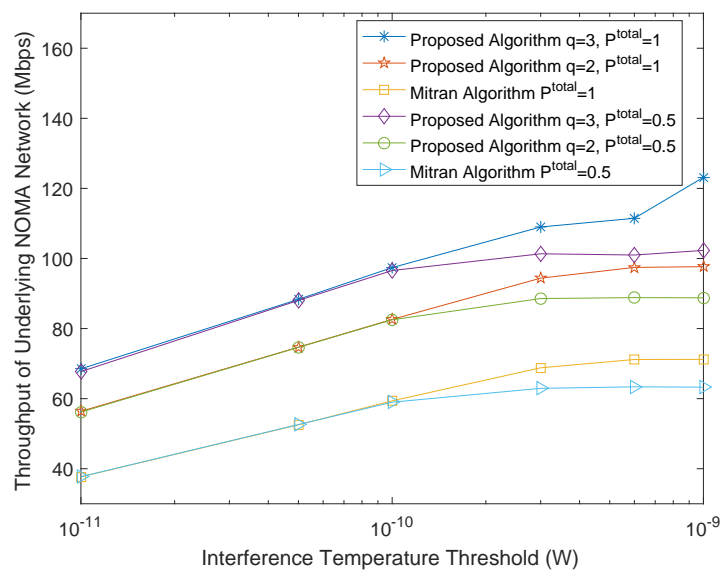

Fig. 3. Total throughput vs. interference temperature threshold imposed by the PUs.

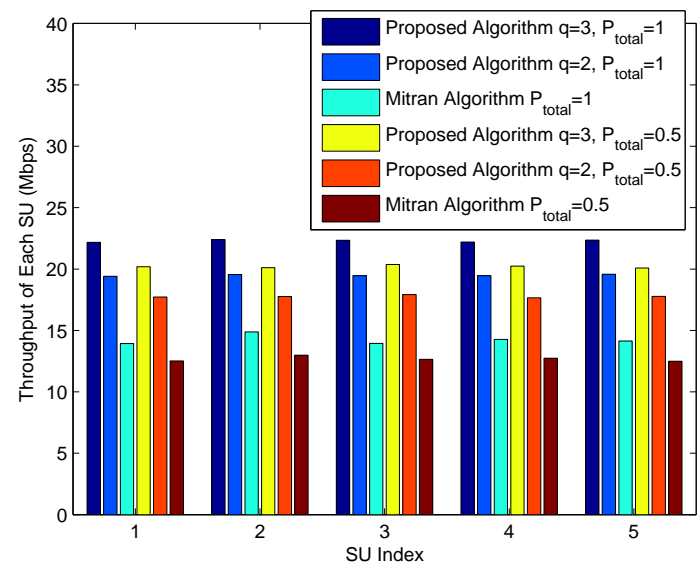

Fig. 4. Average throughput of each SU vs. SU index.

the interference temperature threshold. This is because there is no more power to improve the throughput for SU. The nonsmooth behavior for the the last two points in Fig. 3 is due to the fact that the increase in throughput for the case with 


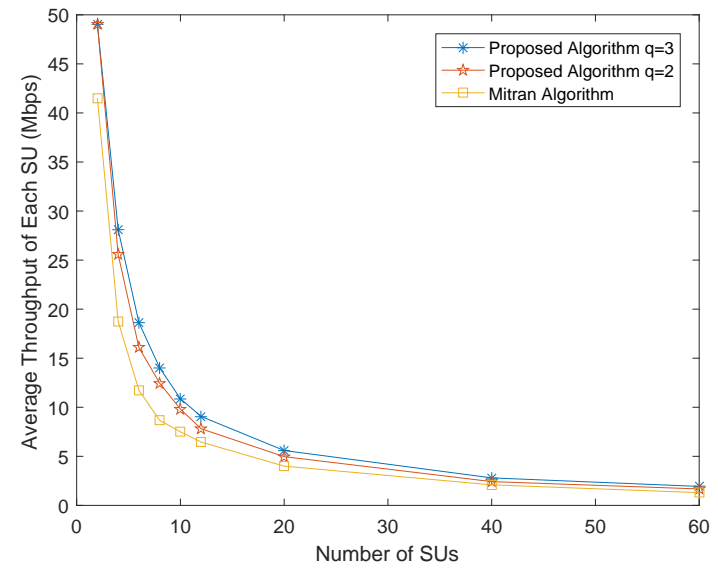

Fig. 5. Average throughput vs. number of SUs.

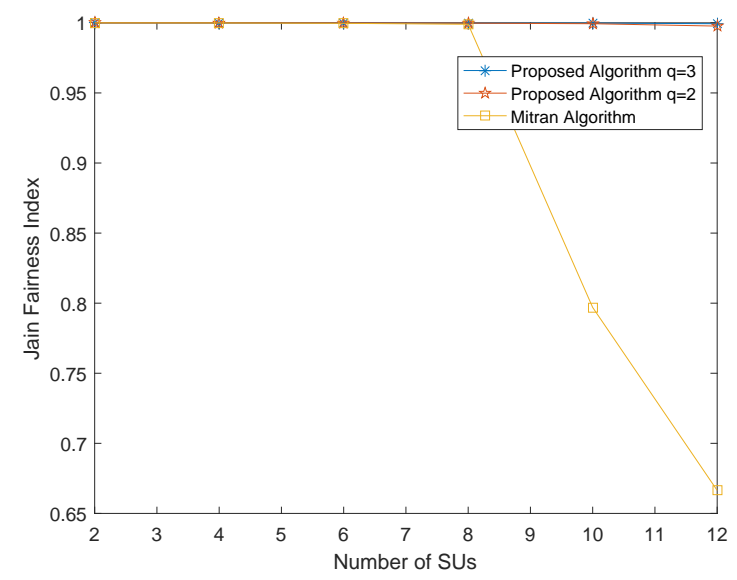

Fig. 6. Jain fairness index vs. number of SUs.

the proposed algorithm with $q_{u}=3$ and $P^{\text {total }}=1 \mathrm{~W}$ is not saturated. The reasons are explained in two folds. Firstly, with the increased total power constraint $P^{\text {total }}=1 \mathrm{~W}$ compared with $P^{\text {total }}=0.5 \mathrm{~W}$, the secondary BS has more power budget for optimal resource allocation. Secondly, with the increased permissive number $q=3$ of NOMA SUs, the multiplex gain yields larger network throughput in total.

We depict the average throughput of each SU versus the SU index in Fig. 4. The simulation parameters are $M=5$ and $K^{v}=16$. There are two cases, i.e., $I_{n}^{\text {th }}=6 \times 10^{-10}$ W. From Fig. 4, we can see that NOMA scheme with $q=3$ can guarantee the proportional fairness very well. Additionally, the throughput for each SU with NOMA scheme is larger than that of Mitran algorithm. Since the proposed algorithm with $q=3$ exploits the power domain diversity more efficiently than that of Mitran algorithm, the proposed algorithm achieves the higher spectrum efficiency than that of Mitran algorithm.

We depict the average throughput versus the number of the SUs in Fig. 5. The simulation parameters are $K^{v}=16$, $I_{n}^{\text {th }}=1 \times 10^{-9} \mathrm{~W}$, and $P^{\text {total }}=1 \mathrm{~W}$. Fig. 5 shows that the average throughput for the three algorithms decrease with increasing the number of SUs. In Fig. 5, as the number of the secondary users increases, more resources are allocated

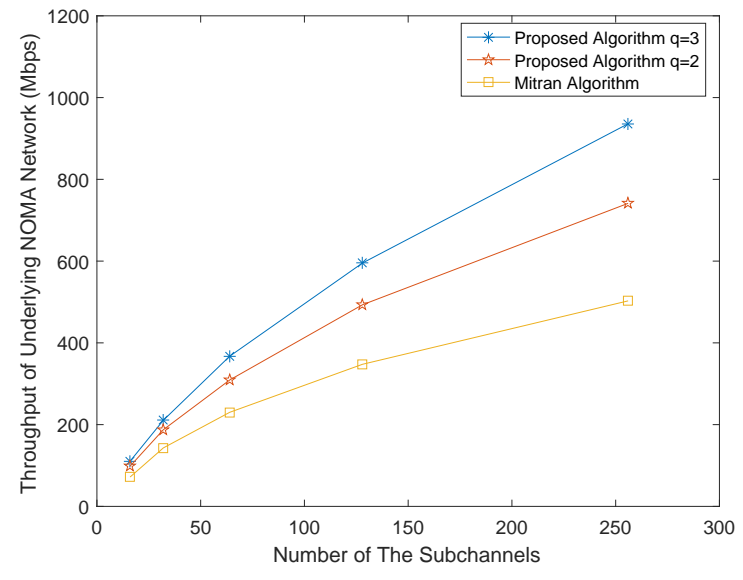

Fig. 7. Total throughput vs. number of subchannels for the underlaying NOMA networks.

to ensure the worst-user throughput among the SUs, and therefore the average throughput decreases when the underlaying NOMA network scales up. Hence, the throughput for each secondary user reduces, and the average throughput for secondary users decreases. This is because the $\mathrm{SU}$, who has the bad CSI, influences the average throughput due to the fairness constraint.

We depict Jain fairness index vs. the number of the SUs for the underlaying NOMA networks in Fig. 6. Jain fairness index is defined in [33]. The simulation parameters are $K^{v}=16$, $I_{n}^{\text {th }}=1 \times 10^{-9} \mathrm{~W}$, and $P^{\text {total }}=1 \mathrm{~W}$. In Fig. 6, it can be observed that the proposed algorithm can guarantee fairness for SUs. However, the fairness of Mitran algorithm becomes bad with the number of SUs due to the lack of spectrum resource.

We depict the throughput versus the number of the subchannels for the underlaying NOMA networks in Fig. 7. The simulation parameters are $M=5, P^{\text {total }}=1 \mathrm{~W}$, and $I_{n}^{\text {th }}=1 \times 10^{-9} \mathrm{~W}$. From Fig. 7, it can be observed that the network throughput for the underlaying NOMA networks increases with the growth of the number of subchannels. This can be explained by the fact that the increased total bandwidth thanks to the growing number of subchannels yields increase in individual SU's transmission rate (cf. (8)) and the network throughput as well. In Fig. 7, we can also observe that the total throughput gap between the proposed algorithm and the Mitran's becomes larger when the number of subchannels grows. This phenomenon shows that the superiority of deploying NOMA other than OMA-based schemes for the underlying secondary networks becomes more significant when the number of sub-channels increases compared with Figs 2 - 5 .

From Figs. 2 to 7, we can conclude that the proposed algorithm improves the fairness among different SUs compared with Mitran algorithm. Since the proposed algorithm at the cost of the receiver complexity improves the network throughput and the secondary network performance, a proper value $q$ can achieve the balance between the receiver complexity and the performance of the underlaying NOMA networks. 


\section{CONCLUding REMARKS}

In this paper, the fairness-aware joint power allocation and SU assignment was studied for an underlaying downlink NOMA network. The formulated problem aimed for maximizing the worst user's throughput subject to the maximum transmitting power at the secondary BS, the cross-channel interference temperature constraints to the PUs in the cognitive network as well as the NOMA constraints. In the proposed alternating optimization based algorithm to solve this MINLP problem, we iteratively solved a subproblem of NOMA-SU assignment and a subproblem NOMA-SU power allocation using a heuristic scheduling algorithm and successive convex approximation, respectively. Results in the numerical examples showed that the proposed algorithm significantly improves not only the worst-user throughput, but also the total and/or the user-average throughput in comparison with the existing scheme in the literature.

This paper investigates the resource allocation with the analog power value feedback. Consequently, the feedback overhead caused by the control channel between the secondary BS and the NOMA SUs may be very high due to the large amount of analog value for the power allocation. This will limit the practical operation for resource allocation algorithm over underlying cognitive NOMA networks. In the possible future directions of this work, to mitigate the costly overhead, we propose to study effective power allocation scheme subject to limited feedback, e.g., by codebook designs for the power values.

\section{Proof OF Proposition 1}

Proof: Define $f(x)=\log _{2}\left(1+2^{x}\right)$, which is a convex function. By first-order Taylor expansion, we know that for each $x_{0}$, we have

$$
\log _{2}\left(1+2^{x}\right)-\log _{2}\left(1+2^{x_{0}}\right) \geq \frac{2^{x_{0}}}{1+2^{x_{0}}}\left(x-x_{0}\right)
$$

Next, we apply the variable transformation of $y=2^{x}$. By substituting $y=2^{x}$ and $y_{0}=2^{x_{0}}$ for $x$ and $x_{0}$ in (30), we can obtain

$\log _{2}(1+y)-\log _{2}\left(1+y_{0}\right) \geq \frac{y_{0}}{1+y_{0}}\left(\log _{2} y-\log _{2} y_{0}\right)$

Now replace $y$ and $y_{0}$ with $\operatorname{SINR}_{m k}$ and $\overline{\operatorname{SINR}}_{m k}, \forall m \in \mathcal{M}$, $k \in \mathcal{K}^{v_{0}}$, respectively, where $\overline{\mathrm{SINR}_{\mathrm{mk}}}$ denotes a constant with the variables $P_{m}^{k}$ and $P_{l}^{k}, l \in \mathcal{M}_{k}^{*}$, in $\mathrm{SINR}_{\mathrm{mk}}$ being fixed as their optimum values in the $j$ th iteration of the SCA method (c.f. Algorithm 2). Consequently, (31) can be rewritten as

$$
\begin{aligned}
& \log _{2}\left(1+\operatorname{SINR}_{m k}\right) \geq \\
& \log _{2}\left(1+\overline{\operatorname{SINR}}_{m k}\right)+ \\
& \frac{\overline{\mathrm{SINR}}_{m k}}{1+\overline{\mathrm{SINR}}_{m k}}\left(\log _{2} \mathrm{SINR}_{m k}-\log _{2} \overline{\operatorname{SINR}}_{m k}\right) .
\end{aligned}
$$

Then, we define

$$
b_{m k}^{\{j\}}=\frac{\overline{\mathrm{SINR}}_{m k}}{1+\overline{\mathrm{SINR}}_{m k}}, \forall m \in \mathcal{M}, k \in \mathcal{K}^{v_{0}}
$$

and

$$
\begin{aligned}
c_{m k}^{\{j\}} & =\log _{2}\left(1+\overline{\mathrm{SINR}}_{m k}\right) \\
& -\frac{\overline{\mathrm{SINR}}_{m k}}{1+\overline{\mathrm{SINR}}_{m k}} \log _{2}\left(\overline{\mathrm{SINR}}_{m k}\right), \forall m \in \mathcal{M}, k \in \mathcal{K}^{v_{0}} .
\end{aligned}
$$

Combining (32)-(34), it follows that

$$
\begin{aligned}
R_{m}^{k} & =\alpha_{m}^{k} B \log _{2}\left(1+\operatorname{SINR}_{m k}\right) \\
& \geq \alpha_{m}^{k} B\left[b_{m k} \log _{2}\left(\operatorname{SINR}_{m k}\right)+c_{m k}\right] .
\end{aligned}
$$

With fixed $b_{m k}$ 's and $c_{m k}$ 's, $R_{m}^{k}$ 's is still non-concave as $P_{m}^{k}$ 's exists in both the nominator and the denominator of SINR $_{m k}$ 's (c.f. (6)), and thus the transformation of $\hat{P}_{m}^{k}=$ $\ln P_{m}^{k}$ is further introduced for the purpose of transforming $\left(\mathrm{OP}^{\prime}\right)$ into a convex problem.

Obviously, the objective function and the constraints in the problem $(\mathrm{OP} 4)$ are concave with variable $\psi$. Consequently, the problem (OP4) is a convex problem with variable $\psi$. Now, we prove problem (OP4) is a convex problem with variable $\hat{P}_{m}^{k}$ 's. According to the objective function and the constraints in the problem (OP4), we define the functions $g_{1}, f_{1}, f_{2}$, and $f_{3}$, i.e.,

$$
\left\{\begin{array}{l}
g_{1}=\psi \\
f_{1}=P^{\text {total }}-\sum_{m \in \mathcal{M}}\left[P_{c}+\sum_{k \in \mathcal{K}^{v_{0}}} \alpha_{m}^{k} \exp \left(\hat{P}_{m}^{k}\right)\right] \\
f_{2}=I_{n}^{t h}-\sum_{m \in \mathcal{M}} \sum_{k \in \mathcal{K}^{v_{0}}} \alpha_{m}^{k} I_{m n}^{k} \exp \left(\hat{P}_{m}^{k}\right), \forall n \in \mathcal{N} \\
f_{3}=\sum_{k \in \mathcal{K}^{v_{0}}} \alpha_{m}^{k} B\left[b_{m k} \log _{2}\left(\exp \left(\hat{P}_{m}^{k}\right) g_{m}^{k}\right)-B_{m k}\right]-\psi .
\end{array}\right.
$$

The second derivatives of $g_{1}, f_{1}, f_{2}$ and $f_{3}$ with respective to $\hat{P}_{m}^{k}$, s are

$$
\left\{\begin{array}{c}
\frac{\partial^{2} g_{1}}{\partial\left(\hat{P}_{m}^{k}\right)^{2}}=0 \leq 0 \\
\frac{\partial^{2} f_{1}}{\partial\left(\hat{P}_{m}^{k}\right)^{2}}=-\alpha_{m}^{k} \exp \left(\hat{P}_{m}^{k}\right) \leq 0 \\
\frac{\partial^{2} f_{2}}{\partial\left(\hat{P}_{m}^{k}\right)^{2}}=-\alpha_{m}^{k} I_{m n}^{k} \exp \left(\hat{P}_{m}^{k}\right) \leq 0, \forall n \in \mathcal{N} \\
\frac{\partial^{2} f_{3}}{\partial\left(\hat{P}_{m}^{k}\right)^{2}}=0 \leq 0, \forall m \in \mathcal{M}
\end{array}\right.
$$

From (37), the objective function and the constraints are concave on $\hat{P}_{m}^{k}$ 's. Additionally, the functions $g_{1}, f_{1}, f_{2}$, and $f_{3}$ are linear functions on the variable $\psi$. Consequently, the problem (OP4) is a convex optimization problem.

\section{PROOF OF LEMMA 1}

Proof: Take the partial derivative of formula (20), i.e.,

$$
\frac{\partial f\left(v, u_{m}, \delta_{n}, \hat{P}_{m}^{k}, \psi\right)}{\partial \hat{P}_{m}^{k}}=0 .
$$

It is equivalent to

$$
\begin{aligned}
& \sum_{m \in \mathcal{M}} \sum_{k \in \mathcal{K}^{v_{0}}}\left(1+u_{m}\right) \alpha_{m}^{k} B b_{m k} / \ln 2 \\
= & \sum_{m \in \mathcal{M}} \sum_{k \in \mathcal{K}^{v_{0}}} \alpha_{m}^{k}\left(v+\sum_{n \in \mathcal{N}} \delta_{n} I_{m n}^{k}\right)
\end{aligned}
$$




\section{REFERENCES}

[1] A. Kliks, O. Holland, A. Basaure, and M. Matinmikko, "Spectrum and license flexibility for $5 \mathrm{G}$ networks," IEEE Comm. Mag., vol. 53, no. 7, pp. 42-49, Jul. 2015.

[2] J. Hansen, D. E. Lucani, J. Krigslund, M. Medard, and F. H. P. Fitzek, "Network coded software defined networking: enabling 5G transmission and storage networks," IEEE Comm. Mag., vol. 53, no. 9, pp. 100-107, Sep. 2015.

[3] A. Benjebbour, A. Li, K. Saito, Y. Saito, Y. Kishiyama, and T. Nakamura, "NOMA: From concept to standardization," in Proc.2015 IEEE CSCN, Oct. 2015, pp. 18-23.

[4] K. Saito, A. Benjebbour, Y. Kishiyama, Y. Okumura, and T. Nakamura, "Performance and design of SIC receiver for downlink NOMA with open-loop SU-MIMO," in Proc.2015 IEEE ICCW, Jun. 2015, pp. 11611165.

[5] L. Lv, J. Chen, and Q. Ni, "Cooperative non-orthogonal multiple access in cognitive radio," IEEE Commun. Lett., vol. 20, no. 10, pp. 2059-2062, Oct. 2016.

[6] L. Lv, J. Chen, Q. Ni, and Z. Ding, "Design of cooperative nonorthogonal multicast cognitive multiple access for $5 \mathrm{G}$ systems: User scheduling and performance analysis," IEEE Trans. Commun., vol. PP, no. 99, pp. 1-1, 2017.

[7] Y. Liu, Z. Ding, M. Elkashlan, and J. Yuan, "Nonorthogonal multiple access in large-scale underlay cognitive radio networks," IEEE Trans. Veh. Tech., vol. 65, no. 12, pp. $10152-10157$, Dec. 2016.

[8] L. Xu, A. Nallanathan, X. Pan, J. Yang, and W. Liao, "Security-aware resource allocation with delay constraint for NOMA-based cognitive radio network," IEEE Trans. Information Forensics and Security, vol. 13, no. 2, pp. 366-376, Feb. 2018.

[9] S. Fu, F. Fang, L. Zhao, Z. Ding, and X. Jian, "Joint transmission scheduling and power allocation in non-orthogonal multiple access," IEEE Trans. Commun., vol. 67, no. 11, pp. 8137-8150, Nov. 2019.

[10] C. He, Y. Hu, Y. Chen, and B. Zeng, "Joint power allocation and channel assignment for NOMA with deep reinforcement learning," IEEE J. Sel. Areas Commun., vol. 37, no. 10, pp. 2200-2210, Oct. 2019.

[11] J. Tang, Y. Yu, M. Liu, D. K. C. So, X. Zhang, Z. Li, and K. Wong, "Joint power allocation and splitting control for SWIPT-enabled NOMA systems," IEEE Trans. Wireless Communications, vol. 19, no. 1, pp. 120-133, Jan. 2020

[12] M. Youssef, J. Farah, C. A. Nour, and C. Douillard, "Resource allocation in NOMA systems for centralized and distributed antennas with mixed traffic using matching theory," IEEE Trans. Commun., vol. 68, no. 1, pp. 414-428, Jan. 2020

[13] J. He, Z. Tang, Z. Tang, H. Chen, and C. Ling, "Design and optimization of scheduling and non-orthogonal multiple access algorithms with imperfect channel state information," IEEE Trans. Veh. Tech., vol. 67, no. 11 , pp. $10800-10814$, Nov. 2018

[14] L. Bai, L. Zhu, Q. Yu, J. Choi, and W. Zhuang, "Transmit power minimization for vector-perturbation based NOMA systems: A sub-optimal beamforming approach," IEEE Trans. Wireless Commun., vol. 18, no. 5, pp. 2679-2692, May 2019.

[15] M. Choi, J. Kim, and J. Moon, "Dynamic power allocation and user scheduling for power-efficient and delay-constrained multiple access networks," IEEE Trans. Wireless Commun., vol. 18, no. 10, pp. 48464858, Oct. 2019.

[16] J. Tang, J. Luo, M. Liu, D. K. C. So, E. Alsusa, G. Chen, K. Wong, and J. A. Chambers, "Energy efficiency optimization for NOMA with SWIPT," IEEE J. Sel. Topics in Sig. Processing, vol. 13, no. 3, pp. 452-466, Jun. 2019.

[17] F. Fang, J. Cheng, and Z. Ding, "Joint energy efficient subchannel and power optimization for a downlink NOMA heterogeneous network," IEEE Trans. Veh. Tech., vol. 68, no. 2, pp. 1351-1364, Feb. 2019.

[18] Z. Yang, W. Xu, Y. Pan, C. Pan, and M. Chen, "Energy efficient resource allocation in machine-to-machine communications with multiple access and energy harvesting for IoT," IEEE Internet Things J., vol. 5, no. 1, pp. 229-245, Feb. 2018.

[19] W. Liang, Z. Ding, Y. Li, and L. Song, "User pairing for downlink nonorthogonal multiple access networks using matching algorithm," IEEE Trans. Commun., vol. 65, no. 12, pp. 5319-5332, Dec. 2017.

[20] Z. Yang, Z. Ding, P. Fan, and N. Al-Dhahir, "A general power allocation scheme to guarantee quality of service in downlink and uplink NOMA systems," IEEE Trans. Wireless Commun., vol. 15, no. 11, pp. 7244 7257, Nov. 2016.

[21] F. Li, H. Jiang, R. Fan, and P. Tan, "Cognitive non-orthogonal multiple access with energy harvesting: An optimal resource allocation approach," IEEE Trans. Veh. Tech., vol. 68, no. 7, pp. 7080-7095, Jul. 2019.
[22] S. Wang, Z. H. Zhou, M. Ge, and C. Wang, "Resource allocation for heterogeneous cognitive radio networks with imperfect spectrum sensing," IEEE J. Sel. Areas Commun., vol. 31, no. 3, pp. 464-475, Mar. 2013.

[23] G. Auer, V. Giannini, C. Desset, I. Godor, P. Skillermark, M. Olsson, M. Imran, D. Sabella, M. Gonzalez, O. Blume, and A. Fehske, "How much energy is needed to run a wireless network?" IEEE Wireless Commun., vol. 18, no. 5, pp. 40-49, Oct. 2011.

[24] A. G. Marques, L. M. Lopez-Ramos, G. B. Giannakis, and J. Ramos, "Resource allocation for interweave and underlay CRs under probabilityof-interference constraints," IEEE J. Sel. Areas Commun., vol. 30, no. 10, pp. 1922-1933, Nov. 2012.

[25] D. T. Ngo, S. Khakurel, and T. Le-Ngoc, "Joint subchannel assignment and power allocation for OFDMA femtocell networks," IEEE Trans. Wireless Commun., vol. 13, no. 1, pp. 342-355, Jan. 2014.

[26] S. Boyd and L. Vandenberghe, Convex Optimization. Cambridge University Press, 2004.

[27] B. R. Marks and G. P. Wright, "Technical note: a general inner approximation algorithm for nonconvex mathematical programs," Oper. Res., vol. 26, no. 4, pp. 681-683, 1978.

[28] Q. Chen, G. Yu, R. Yin, and G. Y. Li, "Energy-efficient user association and resource allocation for multistream carrier aggregation," IEEE Trans. Veh. Technology, vol. 65, no. 8, pp. 6366-6376, Aug. 2016.

[29] J. Papandriopoulos and J. S. Evans, "SCALE: a low-complexity distributed protocol for spectrum balancing in multiuser DSL networks," IEEE Trans. Information Theory, vol. 55, no. 8, pp. 3711-3724, Aug. 2009.

[30] T. Wang and L. Vandendorpe, "On the SCALE algorithm for multiuser multicarrier power spectrum management," IEEE Trans. Signal Process. vol. 60, no. 9, pp. 4992-4998, Sep. 2012.

[31] H. Xing, K. Wong, A. Nallanathan, and R. Zhang, "Wireless powered cooperative jamming for secrecy multi-af relaying networks," IEEE Trans. Wireless Commun., vol. 15, no. 12, pp. 7971-7984, Dec. 2016.

[32] P. Mitran, L. B. Le, and C. Rosenberg, "Queue-aware resource allocation for downlink OFDMA cognitive radio networks," IEEE Trans. Wireless Commun., vol. 9, no. 10, pp. 3100-3111, Oct. 2010.

[33] J. Huang and H. Lu, "Achieving large sum rate and good fairness in MISO broadcast communication," IEEE Trans. Veh. Tech., vol. 68, no. 6 , pp. 5684-5695, Jun. 2019. 